\title{
Efficient Computation of Surface Fields Excited on a Dielectric-Coated Circular Cylinder
}

\author{
Vakur B. Ertürk and Roberto G. Rojas, Senior Member, IEEE
}

\begin{abstract}
An efficient method to evaluate the surface fields excited on an electrically large dielectric-coated circular cylinder is presented. The efficiency of the method results from the circumferentially propagating representation of the Green's function as well as its efficient numerical evaluation along a steepest descent path. The circumferentially propagating series representation of the appropriate Green's function is obtained from its radially propagating counterpart via Watson's transformation and then the path of integration is deformed to the steepest descent path on which the integrand decays most rapidly. Numerical results are presented that indicate that the representations obtained here are very efficient and valid even for arbitrary small separations of the source and field points. This work is especially useful in the moment-method analysis of conformal microstrip antennas where the mutual coupling effects are important.
\end{abstract}

Index Terms-Conformal antennas, electromagnetic coupling, Green function, microstrip arrays.

\section{INTRODUCTION}

M ICROSTRIP antennas and arrays have gained prominence over the last 20 years and have naturally replaced conventional antennas for military as well as commercial applications, ranging from satellite and wireless communications to remote sensing and biomedical applications, due to their low fabrication cost, light weight, mass production, conformity to surface, and direct integrability with other microwave and solid-state devices. Although many practical applications such as high-velocity aircraft, missiles, space vehicles, etc. have stringent aerodynamic constraints that require the use of antennas that conform to their surfaces, the majority of the work for microstrip elements have been for planar structures. This necessitates the development of efficient analytical and numerical tools for this class of antennas conformal to cylindrically shaped substrates. Therefore, the study of surface fields, created by a current distribution on the surface of a material coated perfect electric conducting (PEC) circular cylinder, has been a subject of interest for many years due to its applications in the analysis of conformal microstrip antennas. Furthermore,

Manuscript received August 9, 1999; revised February 16, 2000. This work was supported in part by the U.S. Army Research Office under Grant DAAG55-98-1-0498 and in part by The Ohio State University Research Foundation.

V. B. Erturk was with the ElectroScience Laboratory, Department of Electrical Engineering, The Ohio State University, Columbus, OH 43212-1191 USA. He is now with the Department of Electrical and Electronics Engineering, Bilkent University, Bilkent, Ankara 06533, Turkey (e-mail: vakur@ee.bilkent.edu.tr).

R. G. Rojas is with the ElectroScience Laboratory, Department of Electrical Engineering, The Ohio State University, Columbus, OH 43212-1191 USA (e-mail: rojas@osu.edu)

Publisher Item Identifier S 0018-926X(00)09348-0. it acts as a canonical problem useful toward the development of asymptotic solutions valid for arbitrary smooth coated surfaces.

Early work on the subject of surface wave propagation on curved surfaces was carried out by Wait et al. [1], [2] to study ground wave propagation/attenuation on spherical and cylindrical surfaces satisfying impedance boundary conditions. The study of a small-diameter coated conducting wire supporting surface wave propagation (Goubau line) was carried out in [3] for microwave transmission line applications. More recent work on the derivation of the rigorous dyadic Green's function using a spectral domain representation (radially propagating) for an electric dipole located on the surface of a dielectric coated PEC circular cylinder has been presented in [4] and [5]. Spectral domain Green's functions for coated cylinders and spheres are used in [6] for the design of printed antennas and transmission lines. However, due to the computational complexity of the solutions in [4]-[6], which involve series representations in terms of Bessel and Hankel functions and Fourier integrals, most of the numerical results have been given for electrically small cylinders. It is well known that the spectral representation of the Green's function has convergence problems for large cylinders and separations between source and observation points. This problem can be alleviated to some extent by using carefully chosen basis functions in moment-method-based solutions. Furthermore, the number of terms $(N)$ to be summed in the series increases with the electrical size of the cylinder. This makes the solution intractable, in particular, at high frequencies, where the order of Bessel and Hankel functions as well as their arguments become large resulting in numerical instabilities during the evaluation of the summations/integrations. Nakatini et al. [4] addressed the second problem writing these functions as logarithmic derivatives and calculating these ratios via recurrence relations and the continued fraction method so that high-order Bessel and Hankel functions with large arguments can be evaluated accurately. The dispersion of waves guided along a cylindrical substrate-superstrate layered medium was studied in [7] giving emphasis to the solution of the dispersion equation. Pearson [8] developed integral expressions for the fields of a $z$-directed point source radiating in the presence of a cylindrically layered obstacle as well as asymptotic expressions [9] for source and observation points widely removed from the cylinder. Munk [10] heuristically derived UTD-based Green's functions for the surface fields on a material coated arbitrarily convex conducting surfaces generalizing the asymptotic results of a coated circular cylinder and a coated sphere. His work in [10] includes surface fields up to order $O\left(1 /\left(k_{0} s\right)^{3}\right)$, where $k_{0}$ is the free-space propagation constant and $s$ is the arc length of the geodesic ray path on the surface from the source to the 
observation point. The scheme followed in [10] is an extension of the method developed in [11] and [12] for metallic surfaces. That scheme is a two-step procedure where the leading term $\left(O\left(1 / k_{0} s\right)\right)$ of the potentials $A_{z}$ and $F_{z}$ are first developed and the fields are then obtained by taking the second derivative of $A_{z}$ and $F_{z}$, dropping terms higher than $O\left(1 /\left(k_{0} s\right)^{3}\right)$ and $O(1 / d)$ where $d$ is the radius of the cylinder. That procedure becomes quite complex for dielectric coated surfaces. The surface wave solution in [10] was implemented in [13] and [14] using a combination of Olver's uniform representation and a two-term Debye approximation for the logarithmic derivative of the Hankel functions. Numerical results showed that reasonable results can be obtained for large separations if only terms of $O\left(1 /\left(k_{0} s\right)\right)$ are included. If terms up to $O\left(1 /\left(k_{0} s\right)^{3}\right)$ are used, the results are not as accurate.

In this paper, we present a highly efficient and accurate method to evaluate the surface fields excited by an electric current source located on the surface of a dielectric coated electrically large circular cylinder. The method is based on obtaining a circumferentially propagating ( $\phi$-propagating) series representation of the appropriate Green's function from its radially propagating ( $\rho$-propagating) counterpart and its efficient numerical evaluation along a steepest descent path (SDP) on which the integrand decays most rapidly. In Section II, formulation of the SDP representation of the special Green's function for a dielectric coated circular cylinder is given along with the deformation of the contour of integration which is required to obtain the aferomentioned representation. Section III deals with the numerical evaluation of some special functions that involve Bessel and Hankel functions as well as the numerical evaluation of the integrals. As shown in Section III, a direct integration along the SDP can be performed efficiently using a Gauss-Hermite quadrature to obtain solutions for large and small separations without the need to perform complicated derivatives. The number of terms required in this algorithm decreases with the distance between the source and field points, making it suitable for the analysis of large cylinders as well as large separations. Numerical results are presented in Section IV, which indicate that in contrast to most asymptotic solutions, the results are valid for arbitrary small separations of source and field points. It is important to note that in the limiting case of large separations, this method reduces to the saddle-point integration considered in [13] and [14] (where only the leading term of $O\left(1 /\left(k_{0} s\right)\right)$ is kept). An $e^{j \omega t}$ time dependence is assumed and suppressed throughout this paper.

\section{FORMULATION}

Consider an elementary surface electric current source given by

$$
\mathbf{J}_{e}=\mathbf{P}_{e} \frac{\delta\left(\phi-\phi^{\prime}\right) \delta\left(z-z^{\prime}\right)}{d}
$$

where

$$
\mathbf{P}_{e}=P_{e}^{z} \hat{z}+P_{e}^{\phi} \hat{\phi}
$$

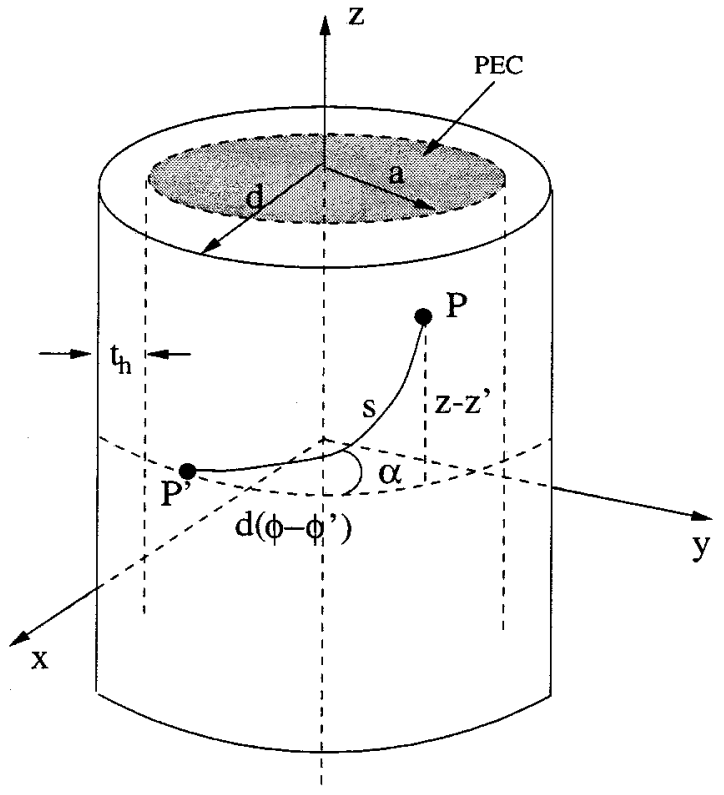

Fig. 1. Dielectric coated PEC circular cylinder where the radius of the PEC cylinder is $a$ and the thickness of the dielectric coating is $t_{h_{t}}=d-a$.

is located on the surface of a dielectric coated circular cylinder whose geometry is given in Fig. $1\left(\rho=\rho^{\prime}=d\right)$. The cylindrical Fourier transform of this current distribution is given by

$$
\tilde{\mathbf{J}}_{e}=\frac{\mathbf{P}_{e}}{2 \pi d} e^{j k_{z} z^{\prime}} e^{j n \phi^{\prime}} .
$$

For such a source defined in (1), the surface fields at $\rho=d$ can be written as

$$
\begin{aligned}
\mathbf{E}(\phi, z)= & \frac{1}{2 \pi} \sum_{n=-\infty}^{\infty} e^{-j n\left(\phi-\phi^{\prime}\right)} \int_{-\infty}^{\infty} \\
& \cdot \frac{\overline{\mathbf{G}} \cdot \mathbf{P}_{e}}{2 \pi d} e^{-j k_{z}\left(z-z^{\prime}\right)} d k_{z}
\end{aligned}
$$

where

$$
\overline{\mathbf{G}}=\overline{\mathbf{G}}\left(n, k_{z}, \rho=\rho^{\prime}=d\right)=\left[\begin{array}{lll}
G_{\rho \rho} & G_{\rho \phi} & G_{\rho z} \\
G_{\phi \rho} & G_{\phi \phi} & G_{\phi z} \\
G_{z \rho} & G_{z \phi} & G_{z z}
\end{array}\right]
$$

is the radially propagating series representation of the appropriate dyadic Green's function. In this paper, we are only interested in the tangential components of the surface fields due to the tangential current sources since most of the momentmethod-based conformal antenna analysis require the use of these components. Therefore, the $\rho$ related components of $\overline{\mathbf{G}}$ (for example $G_{\rho \phi}$ or $G_{z \rho}$, which might be important for applications involving an excitation via a probe) are not taken into consideration (despite the fact that the computation of these components is still the same). Thus, $\overline{\mathbf{G}}_{\tan }$ obtained from (5) is defined as

$$
\overline{\mathbf{G}}_{\mathrm{tan}}=\left[\begin{array}{ll}
G_{\phi \phi} & G_{\phi z} \\
G_{z \phi} & G_{z z}
\end{array}\right]
$$


whose components are explicitly given in [4]. For the sake of clarity, these components are given here again for source and observation points on the surface $\left(\rho=\rho^{\prime}=d\right)$, namely

$$
\begin{aligned}
G_{\phi \phi}= & \frac{j Z_{0}}{k_{0}}\left\{\left[\frac{k_{0}^{2} k_{t 0}}{k_{t 1}^{2}}\right] \frac{R_{n} C_{n}^{e} T_{m}}{T}-k_{t 0} \frac{R_{n} T_{c}^{2}}{\left(\epsilon_{r}-1\right) T}\right. \\
& \left.-\left[\frac{n k_{z}}{d k_{t 1}}\right]^{2} \frac{\left(C_{n}^{e}-k_{t 0} R_{n}\right)}{T}\right\} \\
G_{\phi z}= & \frac{j Z_{0}}{k_{0}}\left[\frac{n k_{z}}{d} \frac{k_{t 0}^{2}}{k_{t 1}^{2}}\right] \frac{\left(C_{n}^{e}-k_{t 0} R_{n}\right)}{T} \\
G_{z \phi}= & G_{\phi z} \\
G_{z z}= & \frac{j Z_{0}}{k_{0}} k_{t 0}^{2} \frac{T_{e}}{T}
\end{aligned}
$$

where

$$
T=T_{e} T_{m}-T_{c}^{2}
$$

with

$$
\begin{aligned}
& T_{e}=k_{t 0} R_{n}-\frac{k_{t 0}^{2}}{k_{t 1}^{2}} C_{n}^{e} \\
& T_{m}=k_{t 0} R_{n}-\epsilon_{r} \frac{k_{t 0}^{2}}{k_{t 1}^{2}} C_{n}^{m} \\
& T_{c}=\frac{k_{0}\left(\epsilon_{r}-1\right)}{k_{t 1}^{2}} \frac{n k_{z}}{d}
\end{aligned}
$$

and

$$
\begin{aligned}
k_{t 0} & =\sqrt{k_{0}^{2}-k_{z}^{2}} ; \quad k_{t 1}=\sqrt{\epsilon_{r} k_{0}^{2}-k_{z}^{2}} \\
R_{n} & =\frac{H_{n}^{(2)}\left(k_{t 0} d\right)}{H_{n}^{(2)}\left(k_{t 0} d\right)} \\
C_{n}^{e} & =k_{t 1} \frac{J_{n}^{\prime}\left(k_{t 1} a\right) Y_{n}^{\prime}\left(k_{t 1} d\right)-J_{n}^{\prime}\left(k_{t 1} d\right) Y_{n}^{\prime}\left(k_{t 1} a\right)}{J_{n}^{\prime}\left(k_{t 1} a\right) Y_{n}\left(k_{t 1} d\right)-J_{n}\left(k_{t 1} d\right) Y_{n}^{\prime}\left(k_{t 1} a\right)} \\
C_{n}^{m} & =k_{t 1} \frac{J_{n}\left(k_{t 1} a\right) Y_{n}^{\prime}\left(k_{t 1} d\right)-J_{n}^{\prime}\left(k_{t 1} d\right) Y_{n}\left(k_{t 1} a\right)}{J_{n}\left(k_{t 1} a\right) Y_{n}\left(k_{t 1} d\right)-J_{n}\left(k_{t 1} d\right) Y_{n}\left(k_{t 1} a\right)}
\end{aligned}
$$

where $k_{0}$ is the free-space wave number, $Z_{0}$ is the free-space intrinsic impedance, and $\left({ }^{\prime}\right)$ denotes derivative with respect to the argument.

It is known that the series in (4) converges very slowly for large cylinders. Moreover, the Green's function involves Bessel and Hankel functions along with their derivatives and their computation for large values of $n$ is not a trivial matter due to the numerical instabilities that occur when the order and argument of these functions become large. Therefore, (4) can be transformed into a more rapidly convergent $\phi$-propagating series representation by using the Watson's transformation. The new series expansion for the fields is given by

$$
\begin{aligned}
\mathbf{E}(\phi, z)= & \frac{1}{4 \pi^{2} d} \int_{-\infty}^{\infty} d k_{z} e^{-j k_{z}\left(z-z^{\prime}\right)} \\
& \cdot\left\{\int_{-\infty-j \epsilon}^{\infty-j \epsilon} \overline{\mathbf{G}}_{\tan }\left(k_{z}, \nu\right)\right.
\end{aligned}
$$

$$
\left.\cdot \mathbf{P}_{e}\left(\sum_{l=-\infty}^{\infty} e^{-j \nu\left(\left(\phi-\phi^{\prime}\right)-2 \pi l\right)}\right) d \nu\right\}
$$

where $\epsilon$ is a small positive number. The expression in (13) can be interpreted as a sum of ray fields that creep $l$ times around the cylinder. Provided that the cylinder is electrically large (a few wavelengths diameter), the first term $(l=0)$ is usually dominant. So, keeping the leading term, (13) can be written as

$$
\begin{aligned}
\mathbf{E}(\phi, z) \approx & \frac{1}{4 \pi^{2} d} \int_{-\infty}^{\infty} d k_{z} e^{-j k_{z}\left(z-z^{\prime}\right)} \\
& \cdot\left\{\int_{-\infty-j \epsilon}^{\infty-j \epsilon} \overline{\mathbf{G}}_{\tan }\left(k_{z}, \nu\right) \cdot \mathbf{P}_{e} e^{-j \nu\left(\phi-\phi^{\prime}\right)} d \nu\right\} .
\end{aligned}
$$

Although (14) converges faster than (4) for electrically large cylinders, computation of the surface fields can be performed more efficiently if the original contour of the $\phi$-propagating representation of the Green's function is deformed into its SDP on which the integrand decays most rapidly. Therefore, making the substitution originally suggested by Fock [15]

$$
\nu=k_{t 0} d+m_{t} \tau
$$

in which

$$
m_{t}=\left(\frac{k_{t 0} d}{2}\right)^{(1 / 3)}
$$

and employing the usual polar transformations

$$
\begin{aligned}
k_{z} & =k_{0} \sin \psi \\
k_{t 0} & =k_{0} \cos \psi
\end{aligned}
$$

along with the geometrical relations based on Fig. 1

$$
\begin{aligned}
z-z^{\prime} & =s \sin \alpha \\
d\left(\phi-\phi^{\prime}\right) & =s \cos \alpha
\end{aligned}
$$

the surface fields can be obtained as

$$
\begin{aligned}
\mathbf{E}(\alpha, s) \approx & \frac{1}{4 \pi^{2} d} \int_{C_{\mathrm{SDP}}} d \psi e^{-j k_{0} s \cos (\alpha-\psi)} \\
& \cdot \int_{C_{\tau}(\psi)} \overline{\mathbf{G}}_{\mathrm{tan}}(\psi, \tau) \cdot \mathbf{P}_{e} e^{-j \xi \tau} d \tau
\end{aligned}
$$

where $s$ is the arc length of the geodesic path on the surface of the coating from the source to the observation point, $\alpha$ is the angle between the ray path and the axis $d\left(\phi-\phi^{\prime}\right)$, and

$$
\xi=m_{t}\left(\phi-\phi^{\prime}\right) \text {. }
$$

In the evaluation of (19), the SDP in the complex $\psi$-plane can be mapped onto the real axis in the $t$-plane by making the substitution

$$
\cos (\alpha-\psi)=1-\frac{j t^{2}}{k_{0} s}
$$

which yields

$$
\mathbf{E}(\alpha, s) \approx \frac{\sqrt{2} e^{j 3 \pi / 4}}{4 \pi^{2} d} \frac{e^{-j k_{0} s}}{\sqrt{k_{0} s}} \cdot \int_{-\infty}^{\infty} d t e^{-t^{2}} F(\alpha, s, t)
$$


with

$$
F(\alpha, s, t)=\frac{1}{\sqrt{1-\frac{j t^{2}}{2 k_{0} s}}} \int_{C_{\tau}(t)} \overline{\mathbf{G}}_{\mathrm{tan}}(t, \tau) \cdot \mathbf{P}_{e} e^{-j \xi \tau} d \tau .
$$

\section{NUMERICAL EVALUATION OF INTEGRALS}

Numerical evaluation of the integrals given in (22) and (23) requires special attention both in the $\tau$-plane and along the SDP (real axis in $t$-plane). Following the procedure given in [13], the integrand $\overline{\mathbf{G}}_{\mathrm{tan}}$ is written in terms of the logarithmic derivative of $H_{\nu}^{(2)}(z)$, namely

$$
R_{\nu}=\frac{H_{\nu}^{(2)^{\prime}}(z)}{H_{\nu}^{(2)}(z)}
$$

with $z=k_{t 0} d$ and $\nu$ is given by (15). Instead of evaluating the Hankel functions and their derivatives separately, the ratio $R_{\nu}$ is evaluated directly to avoid numerical problems and improve its accuracy. These ratios are represented either by a two-term Debye approximation or Olver's uniform representation depending on where these representations are valid and most accurate in the $\tau$-plane. For a two-term Debye approximation [16], $R_{\nu}$ is given by

$$
R_{\nu} \approx-\frac{j \sqrt{z^{2}-\nu^{2}}}{z}-\frac{z}{2\left(z^{2}-\nu^{2}\right)}
$$

whereas, for Olver's uniform representation [16], it is given by

$$
R_{\nu} \approx \frac{A_{i}^{\prime}(x)}{A_{i}(x)} \frac{1}{\nu^{1 / 3}} \frac{e^{j \pi / 3}}{\left[\frac{(z / \nu)^{2} \zeta}{1-(z / \nu)^{2}}\right]^{1 / 2}}
$$

where

$$
\begin{aligned}
x= & \zeta \nu^{2 / 3} e^{-j 2 \pi / 3} \\
\zeta= & (1.5 \rho)^{2 / 3} \\
\rho= & \operatorname{Ln}\left(1+\sqrt{1-(z / \nu)^{2}}\right) \\
& -\operatorname{Ln}(z / \nu)-\sqrt{1-(z / \nu)^{2}}
\end{aligned}
$$

and $A_{i}(x)$ is the Airy function, whereas $A_{i}^{\prime}(x)$ is its derivative with respect to $x$. Olver's uniform representation is used when the two-term Debye approximation fails. Note that use of (26) requires the proper choice of branches in the functions (27a), (27b), and (27c) as explained in [17]. On the other hand, using a two-term Debye approximation for the Bessel functions, analytic closed-form expressions are obtained for the $C_{\nu}^{e}$ and $C_{\nu}^{m}$ functions [18], which are given by

$$
\begin{aligned}
C_{\nu}^{m} \approx & \frac{\sqrt{k_{t 1}^{2}-(\nu / d)^{2}}}{\tan \left(t_{h} \sqrt{k_{t 1}^{2}-(\nu / d)^{2}}\right)}-\frac{1}{2 d} \\
& \cdot\left\{\frac{k_{t 1}^{2}}{k_{t 1}^{2}-(\nu / d)^{2}}-\frac{d^{2}(\nu / d)^{2}}{\sin ^{2}\left(t_{h} \sqrt{k_{t 1}^{2}-(\nu / d)^{2}}\right)}\right\}
\end{aligned}
$$

$$
\begin{array}{r}
C_{\nu}^{e} \approx-\frac{\sqrt{k_{t 1}^{2}-(\nu / d)^{2}}}{\cot \left(t_{h} \sqrt{k_{t 1}^{2}-(\nu / d)^{2}}\right)} \\
+\frac{1}{2 d}\left\{\frac{k_{t 1}^{2} \tan ^{2}\left(t_{h} \sqrt{k_{t 1}^{2}-(\nu / d)^{2}}\right)}{k_{t 1}^{2}-(\nu / d)^{2}}\right. \\
\left.+\frac{d^{2}(\nu / d)^{2}}{\cos ^{2}\left(t_{h} \sqrt{k_{t 1}^{2}-(\nu / d)^{2}}\right)}\right\}
\end{array}
$$

where $t_{h}$ is the thickness of the coating $\left(t_{h}=d-a\right)$. As can be seen, the above expressions provide a very useful and efficient way to calculate the otherwise complicated functions $C_{\nu}^{m}$ and $C_{\nu}^{e}$ since they only involve some elementary functions. Furthermore, these expressions seem to work extremely well for all $k_{z}$ and $\nu$ values even for relatively electrically small cylinders since they are defined as ratios between Bessel functions. Although the approximation for each individual function breaks down, the ratio remains accurate. It is worthwhile to mention at this point that the first terms of (25), (28a), and (28b) can be recognized as the equations corresponding to the planar grounded dielectric slab, whereas the second terms can be treated as the curvature correction terms. Note that there is no branch cut associated with the square roots given in (28a) and (28b). Therefore, the results are independent of the sign chosen for the square roots.

The integral along the real $t$ axis can be easily performed in a very effective and accurate way using a Gauss-Hermite quadrature. The result of this procedure can be written as

$$
\mathbf{E}(\alpha, s) \approx \frac{\sqrt{2} e^{j 3 \pi / 4}}{4 \pi^{2} d} \sum_{q=1}^{Q} F\left(\alpha, s, t_{q}\right) w_{q}
$$

with

$$
F\left(\alpha, s, t_{q}\right)=\frac{e^{-j k_{0} s}}{\sqrt{k_{0} s-\frac{j t_{q}^{2}}{2}}} \int_{C_{\tau}\left(t_{q}\right)} \overline{\mathbf{G}}_{\tan }\left(t_{q}, \tau\right) \cdot \mathbf{P}_{e} e^{-j \xi \tau} d \tau
$$

where $t_{q}$ are the roots of the Hermite polynomials and $w_{q}$ are the appropriate weights. Numerical values for $w_{q}$ and $t_{q}$ can be found in numerical analysis books [16]. In the limiting case where $Q=1, t_{1}=0$ and $w_{1}=\sqrt{\pi}$, this algorithm will recover the leading term of the saddle-point integration considered in [13] and [14], which is valid for large separations between source and observation points.

The integration along the $\tau$-contour is not trivial and a different technique has to be used. First, the integration contour $C_{\tau}(t)$ has to be adjusted for each value of $t_{q}$. This contour mapping is essential because it avoids potential numerical problems due to the $e^{-j \xi \tau}$ term during the integration process and guarantees that no pole crosses the integration contour $C_{\tau}\left(t_{q}\right)$. This task is accomplished by mapping the deformed contour $C$ on the $\nu$ plane, depicted in Fig. 2, onto the $\tau$ plane using $\tau=(\nu-$ $\left.k_{t 0} d / m_{t}\right)\left(\right.$ where $m_{t}=\left(k_{t 0} d / 2\right)^{1 / 3}$ with $k_{t 0}=k_{0} \cos \psi\left(t_{q}\right)$ ) for each $t_{q}$ value. Fig. 3 shows a typical SDP contour on which three $\psi\left(t_{q}\right)$ values are marked and the corresponding $\tau$ contours 


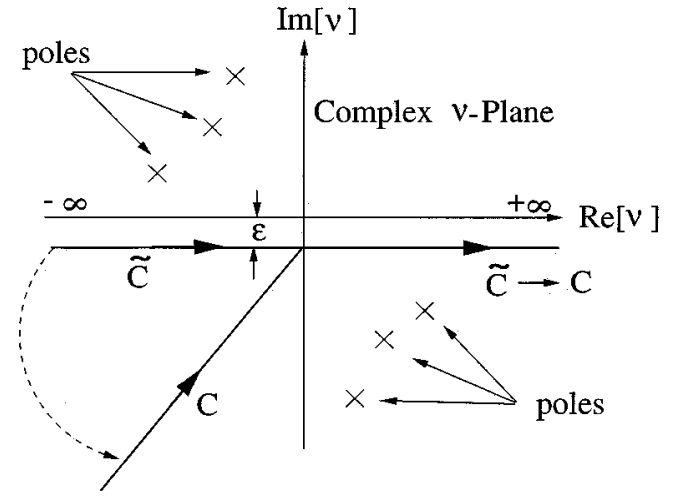

Fig. 2. The original contour of integration $\tilde{C}$ and the deformed contour $C$ in the complex $\nu$ plane.

$\left(C_{\tau}\left(t_{q}\right)\right)$ are illustrated in Fig. 4. Second, the choice of numerical integration algorithm as well as the addition of a proper tail are two important issues due to the oscillatory and slowly decaying nature of the integrands in the $\tau$ domain. In this work, the integration along the $\tau$ contour is performed using Filon's algorithm combined with a Gaussian quadrature. The contour $C_{\tau}\left(t_{q}\right)$ is divided into two regions $C_{\tau}^{-}\left(t_{q}\right)$ as discussed in the Appendix. In region $C_{\tau}^{-}\left(t_{q}\right)$, the integrand decays quickly and the numerical integration can be performed easily. The integration along $C_{\tau}^{+}\left(t_{q}\right)$ is more difficult because the integrand does not decay fast and it is oscillatory. To handle the oscillatory nature of the integrand, Filon's algorithm is used. In this method, part of the integration contour (where the integral is evaluated numerically) is subdivided into half periods determined by $\pi /|\xi|$ to avoid numerical problems that might be encountered if arbitrary intervals are chosen. In the calculation of integrals which contain the $G_{z z}$ and $G_{\phi z}$ (or $G_{z \phi}$ ) components of the dyadic Green's function, the contour $C_{\tau}^{+}\left(t_{q}\right)$ is further divided into two regions where the numerical integration is performed in one region and the second region is integrated analytically. Due to the analytical properties of $G_{\phi \phi}$, the integrals that contain the $G_{\phi \phi}$ component are performed via an envelope extraction technique in the region from 0 to $\infty$ in the $\tau$-domain. In this technique, the asymptotic value of the integrand can be integrated in closed form; therefore, if one subtracts the asymptotic value from the integrand, the resulting integrand is relatively smooth and fast decaying so that it can be integrated efficiently. The analytical details for this case are also given in the Appendix.

\section{NUMERICAL RESULTS}

To access the accuracy of this method, some numerical results for the mutual impedance between two tangential electric current modes are obtained using (29) and compared with the traditional eigenfunction solution given by (4) for a large cylinder with $a=3 \lambda_{0}, t_{h}=0.06 \lambda_{0}, \epsilon_{r}=3.25\left(\lambda_{0}=\right.$ free-space wavelength) and a smaller one with $a=1.5 \lambda_{0}, t_{h}=$ $0.06 \lambda_{0}, \epsilon_{r}=3.25$. The current modes are defined by a piecewise sinusoid along the direction of the current and by constant along the direction perpendicular to the current. Each element has dimensions of $0.05 \lambda_{0}$ (along the direction of the current) by $0.02 \lambda_{0}$. This particular choice of current modes guarantees the convergence of the reference spectral-domain solution (4)

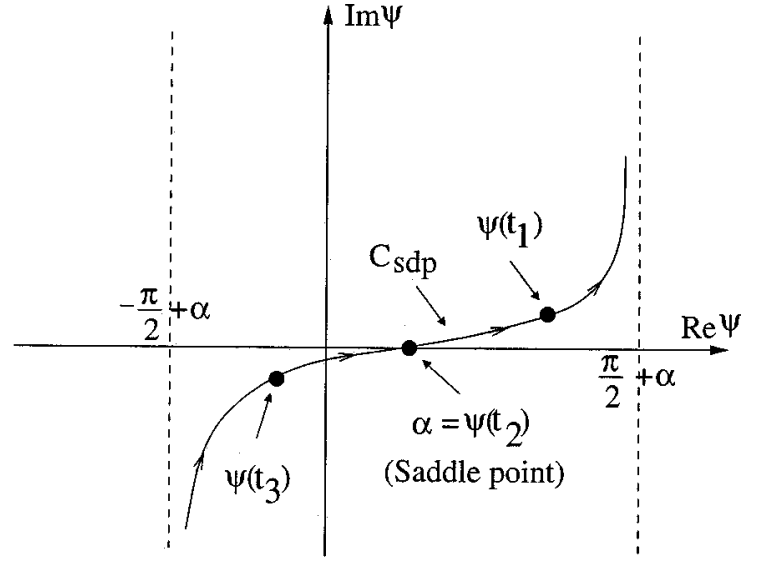

Fig. 3. SDP contour.

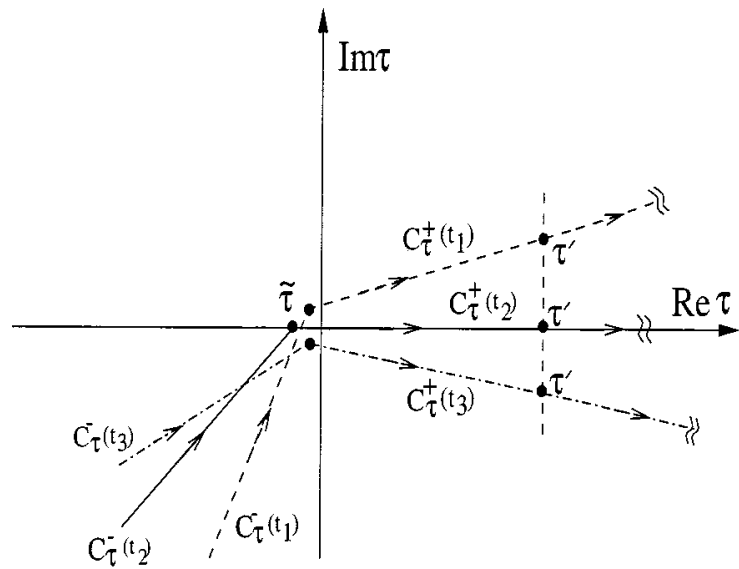

Fig. 4. Typical integration contours in the $\tau$ domain.

for large cylinders, even though the rate of convergence is very slow. Figs. 5-7 show the real and imaginary parts of the mutual impedance between two $z$-directed, a $z$ - and a $\phi$-directed and two $\phi$-directed current sources, respectively, versus separation. The angle $(\alpha)$ for these examples is chosen to be $55^{\circ}, 45^{\circ}$, and $40^{\circ}$, respectively. Similarly, the same type of results are depicted in Figs. 8-10, respectively, for the smaller cylinder where values for $\alpha$ are chosen to be $40^{\circ}, 25^{\circ}$, and $30^{\circ}$. For the smaller cylinder, the effects of multiple wave encirclements around the coated cylinder become visible for separations larger than $3.5 \lambda_{0}$ (or $3 \lambda_{0}$ depending upon the polarization) and, hence, the addition of the $l=|1|$ term given by (13) is necessary. As seen from the figures, excellent agreement is achieved even for separations as small as $0.2 \lambda_{0}$ (even $0.1 \lambda_{0}$ for some cases).

\section{DisCUSSIONS AND CONCLUSION}

A highly efficient and accurate scheme for the evaluation of surface fields excited by electric current sources mounted on an electrically large dielectric-coated circular cylinder is developed. The numerical results obtained from the $\phi$-representation-SDP integration method agree well with the conventional eigenfunction solution, even for electrically small separations between the source and observation points. This is in contrast to most asymptotic solutions considered in the literature where higher order terms [up to $O\left(1 /\left(k_{0} s\right)^{3}\right)$ ] need to be included to 


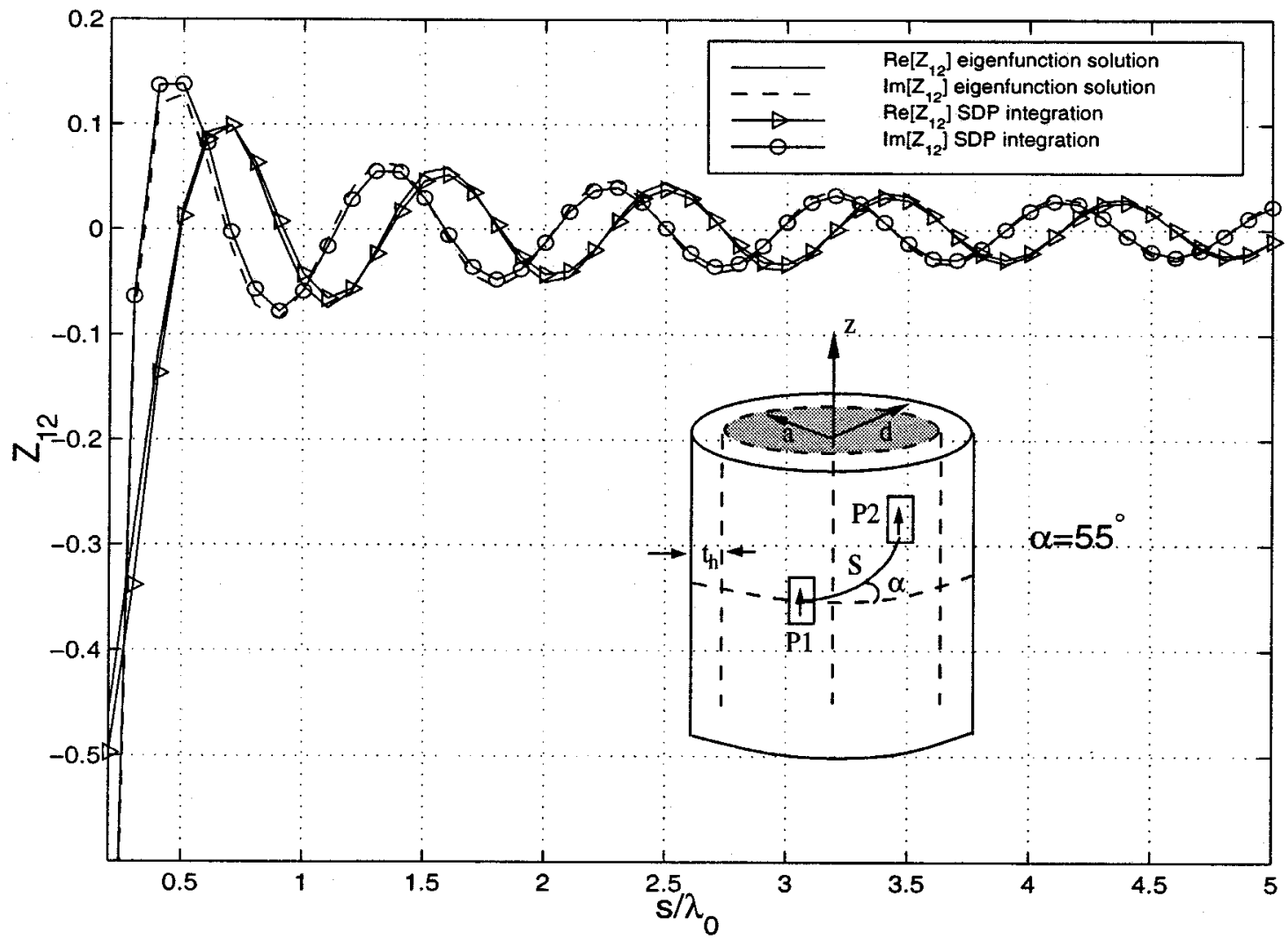

Fig. 5. Real and imaginary parts of the mutual impedance between two identical $z$-directed current sources for a coated cylinder with $a=3 \lambda_{0}, t_{t_{t}}=0.06 \lambda_{0}$, $\epsilon_{r}=3.25 . Z_{12}=\int \mathbf{E}^{(1)}-\mathbf{J}^{(2)} d_{3}$, where $\mathbf{J}^{(2)}$ is source $P_{2}$ and $\mathbf{E}^{(1)}$ is the field due to source $P_{1}$.

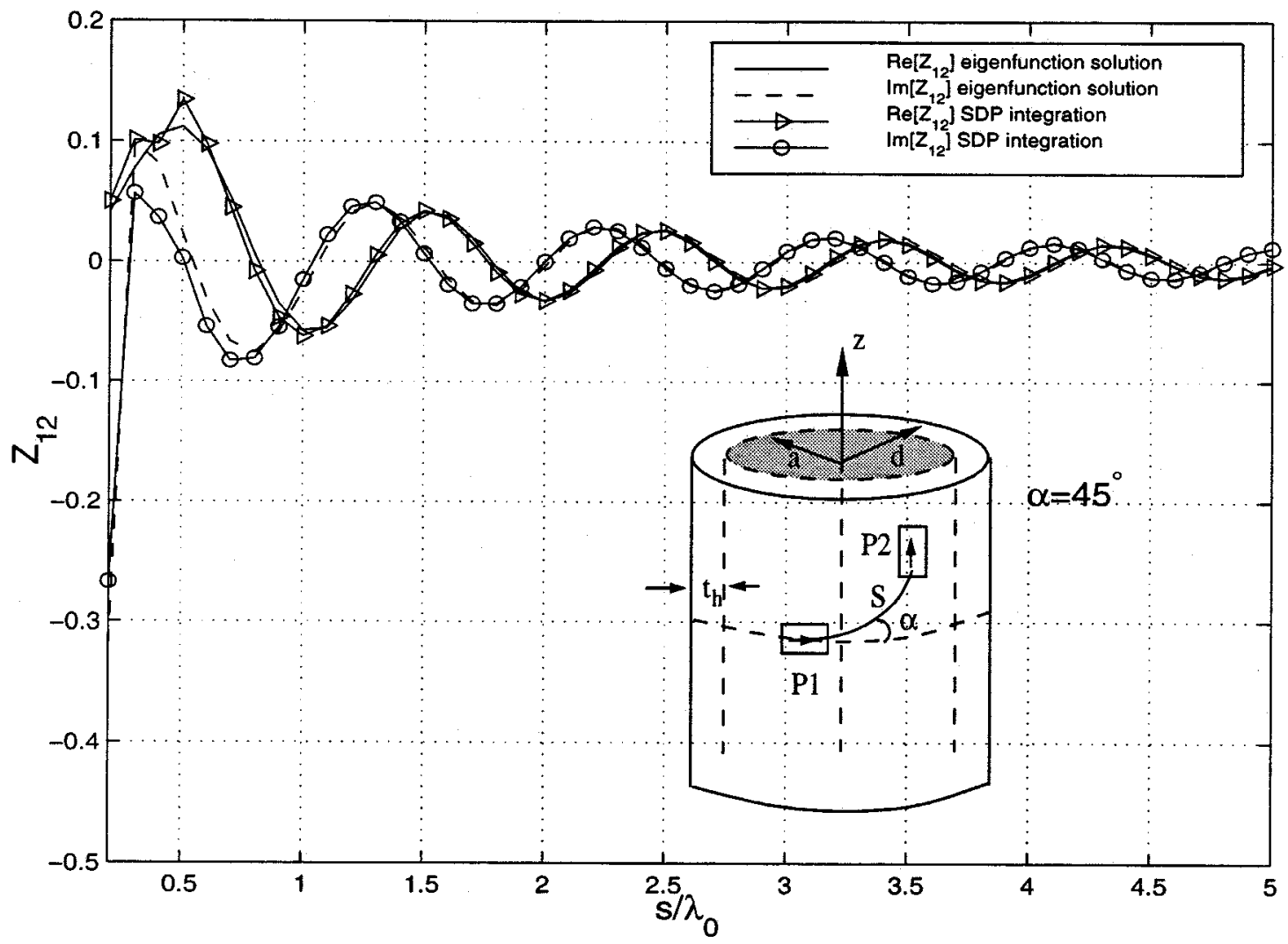

Fig. 6. Real and imaginary parts of the mutual impedance between $z$-and $\phi$-directed current sources for a coated cylinder with $a=3 \lambda_{0}, t_{h}=0.06 \lambda_{0}$, $\epsilon_{r}=3.25$. 


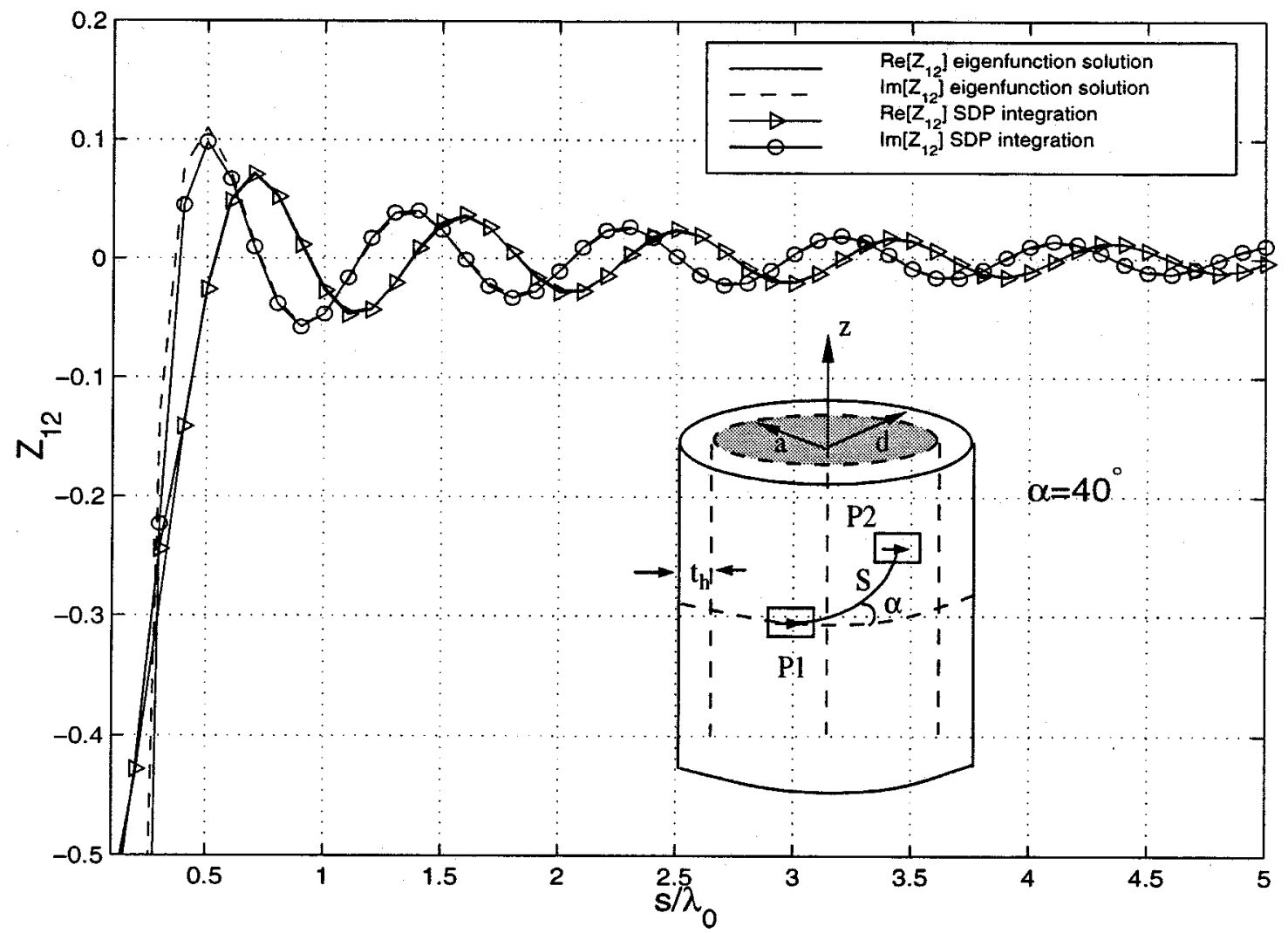

Fig. 7. Real and imaginary parts of the mutual impedance between two identical $\phi$-directed current sources for a coated cylinder with $a=3 \lambda_{0}, t_{h}=0.06 \lambda_{0}$, $\epsilon_{r}=3.25$.

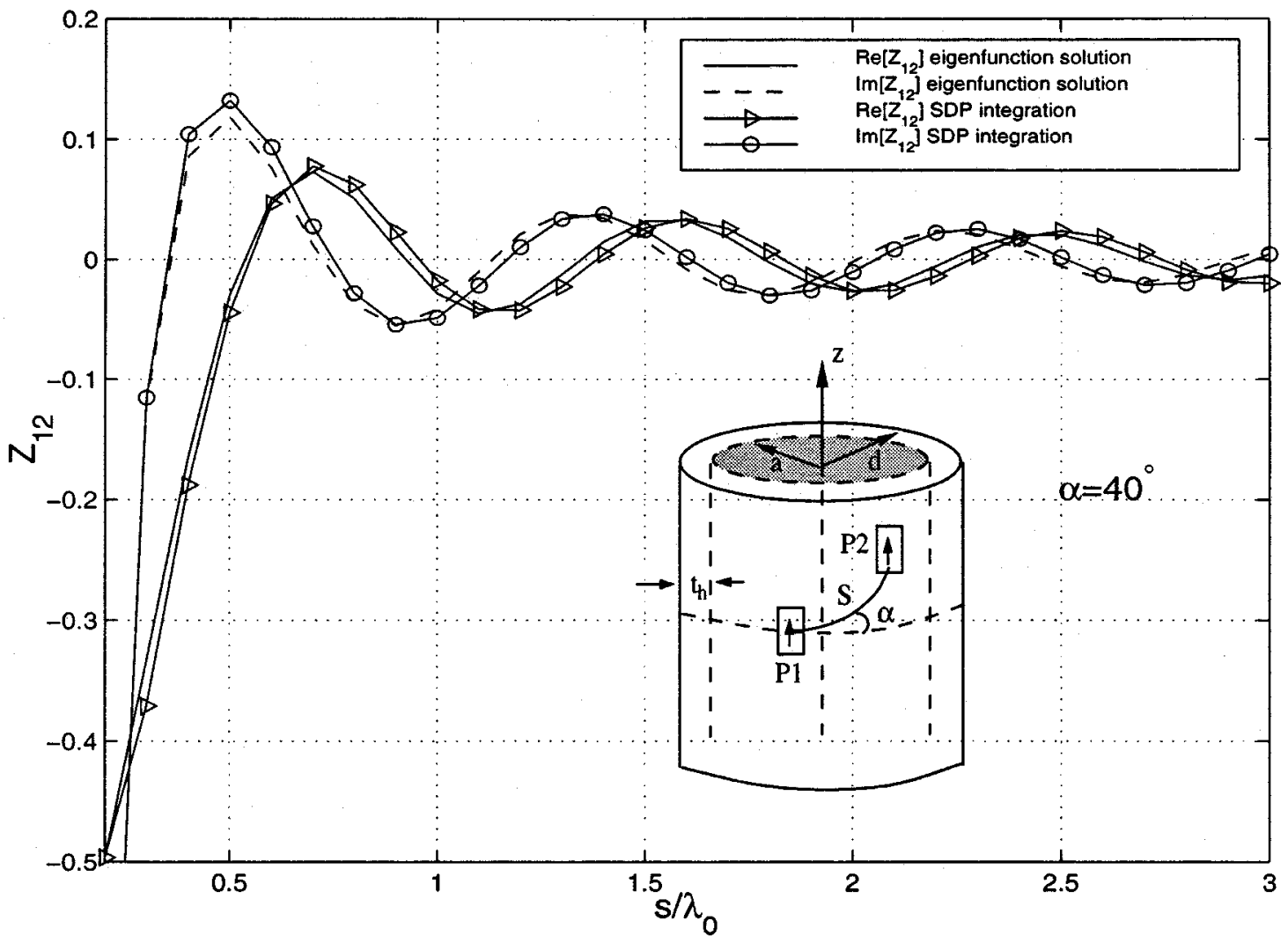

Fig. 8. Real and imaginary parts of the mutual impedance between two identical $z$-directed current sources for a coated cylinder with $a=1.5 \lambda_{0}, t_{h_{t}}=0.06 \lambda_{0}$, $\epsilon_{r}=3.25$ 


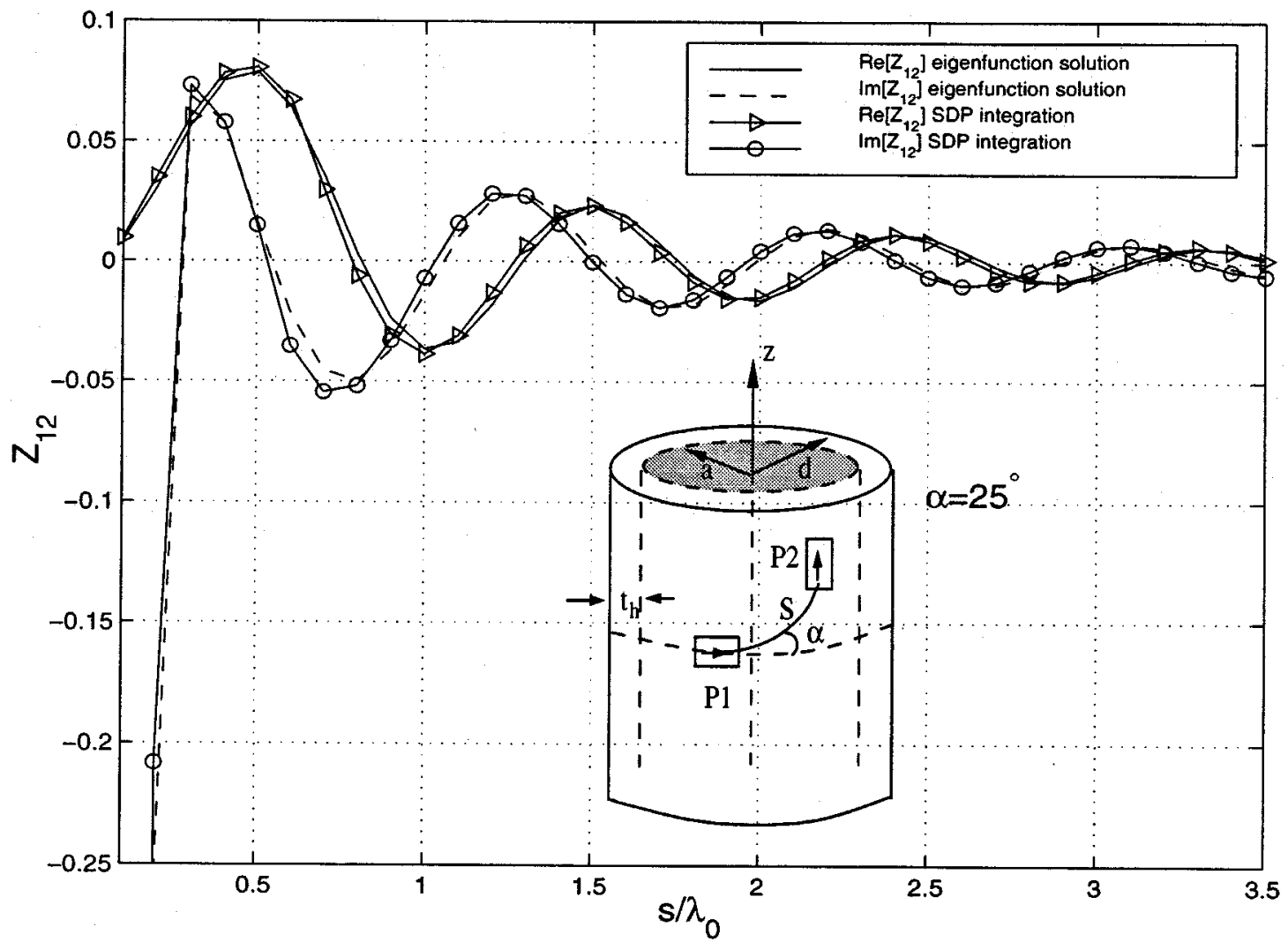

Fig. 9. Real and imaginary parts of the mutual impedance between $z$ - and $\phi$-directed current sources for a coated cylinder with $a=1.5 \lambda_{0}, t_{h}=0.06 \lambda_{0}$, $\epsilon_{r}=3.25$.

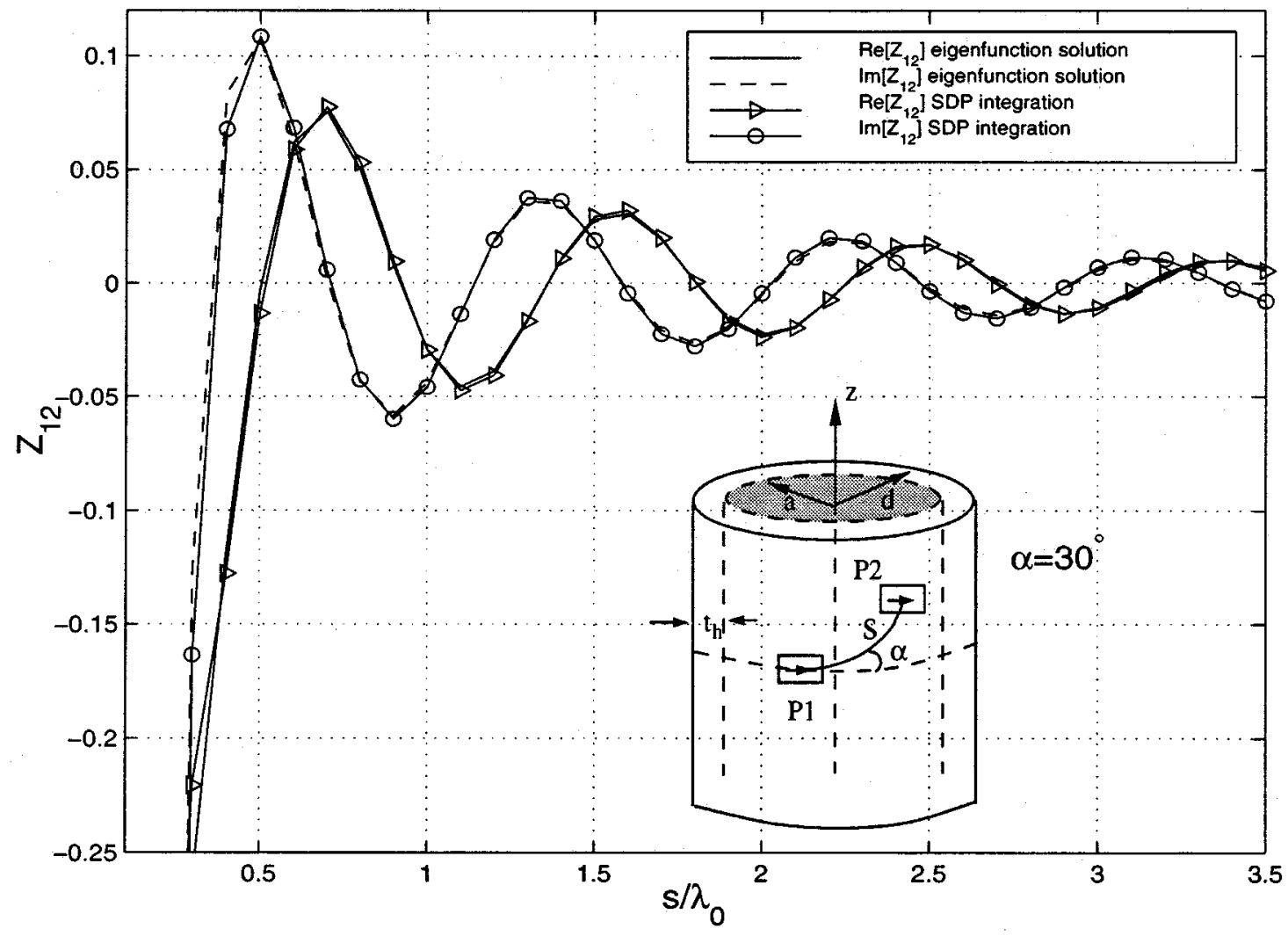

Fig. 10. Real and imaginary parts of the mutual impedance between two identical $\phi$-directed current sources for a coated cylinder with $a=1.5 \lambda_{0}, t_{h}=0.06 \lambda_{0}$, $\epsilon_{r}=3.25$. 
evaluate the surface fields for small separations on PEC or material-coated PEC circular cylinders. However, obtaining these higher order terms is very complicated for a material-coated cylinder. On the other hand, the SDP integration used here allows one to calculate the asymptotic form of the surface fields directly, even for arbitrarily small separations between the source and observation points without the need to take complicated derivatives. However, the solution has some accuracy problems near the paraxial region $(\alpha \longrightarrow \pi / 2)$ of the cylinder due to the mapping given by (15). This is a well-known problem that has been observed for PEC and impedance cylinders in the past, where the mapping in (15) was used. Valid solutions in the paraxial region have also been developed by these authors and will be reported in a separate paper. Nevertheless, the present SDP representation of the Green's function can be used in conjunction with the method of moments to analyze and design arbitrarily shaped conformal antennas on coated cylinders, except for the calculation of the self terms and mutual coupling between two current modes that lie in the paraxial region of the cylinder. The self-term calculations can be carried out using conventional techniques (eigenfunction solution) or assuming that the current element lies on a planar substrate (planar approximation). Furthermore, uniform theory of diffraction (UTD)-based solutions for dielectric coated arbitrarily convex surfaces can be heuristically developed generalizing this solution and the UTD solution of a sphere via the local properties of electromagnetic wave propagation at high frequencies as demonstrated in [10].

\section{APPENDIX}

The limiting values of the Green's function components for large $\tau$ values are given by

$$
\begin{aligned}
\lim _{\tau \rightarrow \infty} G_{z z}\left(t_{q}, \tau\right) & =\frac{B_{1}}{\tau} \\
\lim _{\tau \rightarrow \infty} G_{\phi z}\left(t_{q}, \tau\right) & =B_{2} \\
\lim _{\tau \rightarrow \infty} G_{\phi \phi}\left(t_{q}, \tau\right) & =B_{3} \tau+B_{4}
\end{aligned}
$$

where $B_{i}$ are constants whose values are given by

$$
\begin{aligned}
B_{1}= & \frac{j Z_{0}}{k_{0}} k_{t 0}^{2} \\
& \cdot \frac{\left[1+\frac{k_{t 0}^{2}}{k_{t 1}^{2}}\right]}{\left(\frac{m_{t}}{d}\right)\left[\left(1+\epsilon_{r} \frac{k_{t 0}^{2}}{k_{t 1}^{2}}\right)\left(1+\frac{k_{t 0}^{2}}{k_{t 1}^{2}}\right)-\left(\frac{k_{0}\left(\epsilon_{r}-1\right) k_{z}}{k_{t 1}^{2}}\right)^{2}\right]} \\
B_{2}= & \frac{j Z_{0}}{k_{0}} \frac{k_{t 0}^{2}}{k_{t 1}^{2}} \\
& \cdot \frac{\left.2 k_{z} \mathrm{a}\right)}{\left[\left(1+\epsilon_{r} \frac{k_{t 0}^{2}}{k_{t 1}^{2}}\right)\left(1+\frac{k_{t 0}^{2}}{k_{t 1}^{2}}\right)-\left(\frac{k_{0}\left(\epsilon_{r}-1\right) k_{z}}{k_{t 1}^{2}}\right)^{2}\right]}
\end{aligned}
$$

$$
\begin{aligned}
B_{3}= & \frac{j Z_{0}}{k_{0}} \frac{m_{t}}{d} \\
& \cdot \frac{\left[\frac{k_{0}^{2}}{k_{t 1}^{2}}\left(1+\epsilon_{r} \frac{k_{t 0}^{2}}{k_{t 1}^{2}}\right)+\frac{k_{0}^{2}\left(\epsilon_{r}-1\right) k_{z}^{2}}{k_{t 1}^{4}}-2 \frac{k_{z}^{2}}{k_{t 1}^{2}}\right]}{\left[\left(1+\epsilon_{r} \frac{k_{t 0}^{2}}{k_{t 1}^{2}}\right)\left(1+\frac{k_{t 0}^{2}}{k_{t 1}^{2}}\right)-\left(\frac{k_{0}\left(\epsilon_{r}-1\right) k_{z}}{k_{t 1}^{2}}\right)^{2}\right]} \\
B_{4}= & \frac{j Z_{0}}{k_{0}} k_{t 0} \\
& \cdot \frac{\left[\frac{k_{0}^{2}}{k_{t 1}^{2}}\left(1+\epsilon_{r} \frac{k_{t 0}^{2}}{k_{t 1}^{2}}\right)+\frac{k_{0}^{2}\left(\epsilon_{r}-1\right) k_{z}^{2}}{k_{t 1}^{4}}-2 \frac{k_{z}^{2}}{k_{t 1}^{2}}\right]}{\left[\left(1+\epsilon_{r} \frac{k_{t 0}^{2}}{k_{t 1}^{2}}\right)\left(1+\frac{k_{t 0}^{2}}{k_{t 1}^{2}}\right)-\left(\frac{k_{0}\left(\epsilon_{r}-1\right) k_{z}}{k_{t 1}^{2}}\right)^{2}\right]}
\end{aligned}
$$

Consequently, an integral related with the $G_{z z}$ component of the dyadic Green's function, which is in the form of

$$
I_{1}=C_{1} \int_{C_{\tau}\left(t_{q}\right)} G_{z z}\left(t_{q}, \tau\right) P_{e}^{z} e^{-j \xi \tau} d \tau
$$

can be written as

$$
\begin{aligned}
I_{1}=C_{1} & {\left[\int_{C_{\tau}^{-}\left(t_{q}\right)+C_{\tau}^{+}\left(t_{q}\right)} G_{z z}\left(t_{q}, \tau\right) P_{e}^{z} e^{-j \xi \tau} d \tau\right.} \\
& \left.+\int_{\tau^{\prime}}^{\infty} \frac{B_{1}}{\tau} P_{e}^{z} e^{-j \xi \tau} d \tau\right]
\end{aligned}
$$

where

$$
\int_{\tau^{\prime}}^{\infty} \frac{B_{1}}{\tau} e^{-j \xi \tau} d \tau \approx B_{1} \frac{e^{-j \xi \tau^{\prime}}}{j \xi \tau^{\prime}}
$$

In (34), $C_{\tau}^{-}\left(t_{q}\right)$ is the part of the integration contour on which $\operatorname{Re}(\tau)<\tilde{\tau}$ whereas, $C_{\tau}^{+}\left(t_{q}\right)$ is the part on which $\tilde{\tau}<\operatorname{Re}(\tau)<$ $\operatorname{Re}\left(\tau^{\prime}\right)$. Integrals on these portions are performed numerically as mentioned in Section III. On the other hand, the complex exponential integral (35) is evaluated using a first-order stationary phase method in which only the end-point contributions are considered since the interval $\operatorname{Re}\left(\tau^{\prime}\right)<\operatorname{Re}(\tau)<\infty$ does not contain a stationary point. Furthermore, the contribution from $\infty$ is omitted as mentioned in [19]. Similarly, an integral related with the $G_{\phi z}$ (or $G_{z \phi}$ ) component can be evaluated the same way except that the tail contribution is given by

$$
\int_{\tau^{\prime}}^{\infty} B_{2} e^{-j \xi \tau} d \tau \approx B_{2} \frac{e^{-j \xi \tau^{\prime}}}{j \xi}
$$

However, an integral with the $G_{\phi \phi}$ component is written as

$$
\begin{aligned}
I_{2}=C_{1} & {\left[\int_{C_{\tau}\left(t_{q}\right)} G_{\phi \phi}\left(t_{q}, \tau\right) P_{e}^{\phi} e^{-j \xi \tau} d \tau\right.} \\
& +\int_{0}^{\infty}\left(G_{\phi \phi}\left(t_{q}, \tau\right)-B_{3} \tau-B_{4}\right) P_{e}^{\phi} e^{-j \xi \tau} d \tau \\
& \left.+\int_{0}^{\infty} B_{3} \tau P_{e}^{\phi} e^{-j \xi \tau} d \tau+\int_{0}^{\infty} B_{4} P_{e}^{\phi} e^{-j \xi \tau} d \tau\right] .
\end{aligned}
$$


As a result of this process, the integrand of the second integral also becomes rapidly convergent and can be performed numerically as mentioned in Section III. The result of the fourth integral is given by (36) except $B_{2}$ is replaced by $B_{4}$. Finally, the third integral can be recognized as the Fourier transform of a ramp function and is given by

$$
\int_{0}^{\infty} B_{3} \tau e^{-j \xi \tau} d \tau=-B_{3} \frac{1}{\xi^{2}}
$$

\section{REFERENCES}

[1] J. R. Wait, Electromagnetic Radiation from Cylindrical Structures. New York: Pergamon, 1959.

[2] D. A. Hill and J. R. Wait, "Ground wave attenuation function for a spherical earth with arbitrary surface impedance," Radio Sci., vol. 15, pp. 637-643, May-June 1980.

[3] G. Goubau, "Surface waves and their applications to transmission lines," J. Appl. Phys., vol. 21, pp. 1119-1128, Nov. 1950.

[4] A. Nakatini, N. G. Alexopoulus, N. K. Uzunoglu, and P. L. E. Uslenghi, "Accurate Green's function computation for printed circuit antennas on cylindrical antennas," Electromagn., vol. 6, pp. 243-254, July-Sept. 1986.

[5] T. M. Habashy, S. M. Ali, and J. A. Kong, "Input impedance and radiation pattern of cylindrical-rectangular and wraparound microstrip antennas," IEEE Trans. Antennas Propagat., vol. 38, pp. 722-731, May 1990.

[6] K.-L. Wong, Design of Nonplanar Microstrip Antennas and Transmission Lines. New York: Wiley, 1999.

[7] K. Naishadham and L. B. Felsen, "Dispersion of waves guided along a cylindrical substrate-superstrate layered medium," IEEE Trans. Antennas Propagat., vol. 41, pp. 304-313, Mar. 1993.

[8] L. W. Pearson, "A construction of the fields radiated by a $z$-directed point sources of current in the presence of a cylindrically layered obstacle," Radio Sci., vol. 21, pp. 559-569, July-Aug. 1986.

[9] L. W. Pearson, "A ray representation of surface diffraction by a multilayer cylinder," IEEE Trans. Antennas Propagat., vol. AP-35, pp. 698-707, June 1987.

[10] P. Munk, "A uniform geometrical theory of diffraction for the radiation and mutual coupling associated with antennas on a material coated convex conducting surface," Ph.D. dissertation, Dept. Elect. Eng., Ohio State Univ., Columbus, 1996

[11] P. H. Pathak and R. G. Kouyoumjian, "An analysis of the radiation from apertures in curved surfaces by the geometrical theory of diffraction," Proc. IEEE, vol. 62, pp. 1438-1461, Nov. 1974.

[12] P. H. Pathak and N. Wang, "An analysis of the mutual coupling between antennas on a smooth convex surface," ElectroSci. Lab., Dept. Elect. Eng., Ohio State Univ., Tech. Rep. 784 538-7, Oct. 1978.

[13] C. Demirdag and R. G. Rojas, "Mutual coupling calculations on a dielectric coated PEC cylinder using UTD-based Green's function," in IEEE Antennas Propagat. Symp. Dig., vol. 3, Canada, July 1997, pp. $1525-1528$.

[14] R. G. Rojas and V. B. Ertürk, "UTD ray analysis of mutual coupling and radiation for antennas mounted on dielectric coated PEC convex surfaces," Proc. URSI Int. Symp. Electromagn. Theory, vol. 1, pp. 178-180, May 1998.

[15] V. A. Fock, "Diffraction of radio waves around the earth's surface," $J$. Phys. USSR, vol. 9, pp. 256-266, 1945.

[16] M. Abramowitz and I. A. Stegun, Handbook of Mathematical Functions. New York: Dover, 1970.

[17] R. Paknys, "Evaluation of hankel functions with complex argument and complex order," IEEE Trans. Antennas Propagat., vol. 40, pp. 569-578, May 1992.

[18] M. Marin and P. Pathak, "Calculation of surface fields created by a current distribution on a coated circular cylinder," ElectroSci. Lab., Dept. Elect. Eng., Ohio State Univ., Tech. Rep. 721 565-1, Apr. 1989.

[19] L. B. Felsen and N. Marcuvitz, Radiation and Scattering of Waves. Englewood Cliffs, NJ: Prentice-Hall, 1973.

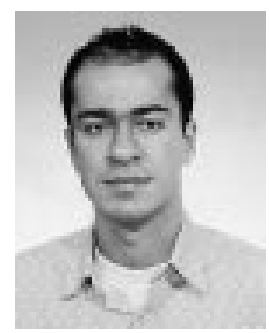

and curved surfaces.
Vakur B. Ertürk received the B.S. degree in electrical engineering from Middle East Technical University, Ankara, Turkey, in 1993 and the M.S. and $\mathrm{Ph} . \mathrm{D}$. degrees from The Ohio State University (OSU), Columbus, in 1996 and 2000, respectively.

During his stay at OSU, he was a Graduate Research Associate at the ElectroScience Laboratory. $\mathrm{He}$ is now with the Department of Electrical Engineering, Bilkent University, Ankara, Turkey. His research interests include design and analysis of active and passive microstrip antennas and arrays on planar

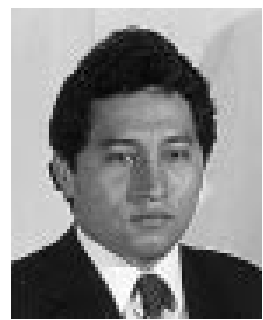

Roberto G. Rojas (S'80-M'85-SM'90) received the B.S.E.E. degree from New Mexico State University, Las Cruces, in 1979 and the M.S. and Ph.D. degrees in electrical engineering from The Ohio State University, Columbus, in 1981 and 1985, respectively.

$\mathrm{He}$ is currently an Associate Professor in the Department of Electrical Engineering, The Ohio State University. His current research interests are the development of analysis and design tools for conformal arrays, active integrated antennas, design of large arrays, as well as the analysis of electromagnetic radiation and scattering phenomena in complex environments.

Dr. Rojas received the 1988 R.W.P. King Prize Paper Award and the 1990 Browder J. Thompson Memorial Prize Award, both given by IEEE, and the 1989 and 1993 Lumley Research Awards, given by the College of Engineering, The Ohio State University. He has served as Chairman, Vice-Chairman, and Secretary/Treasurer of the Columbus, OH, chapter of the IEEE Antennas and Propagation and Microwave Theory and Techniques Societies. He is an elected member of the United States Commission B of URSI. 